\author{
Gian Maria D'Amici' ${ }^{1 *}$ \\ Anna Maria Timperio ${ }^{1 *}$ \\ Federica Gevi ${ }^{1}$ \\ Giuliano Grazzini ${ }^{2}$ \\ Lello Zolla ${ }^{1}$ \\ ${ }^{1}$ Department of Environmental \\ Sciences, Tuscia University, \\ Viterbo, Italy \\ ${ }^{2}$ Italian National Blood Centre, \\ Istituto Superiore di Sanità, \\ Rome, Italy
}

Received April 14, 2010

Revised May 14, 2010

Accepted May 25, 2010
Research Article

\section{Recombinant clotting factor VIII concentrates: Heterogeneity and high-purity evaluation}

\begin{abstract}
Factor VIII is an important glycoprotein involved in hemostasis. Insertion of expression vectors containing either the full-length cDNA sequence of human factor VIII (FLrFVIII) or B-domain deleted (BDDrFVIII) into mammalian cell lines results in the production of recombinant factor VIII (rFVIII) for therapeutic usage. Three commercially available rFVIII concentrates (Advate ${ }^{\circledR}$, Helixate NexGen ${ }^{\mathbb{R}}$ and Refacto ${ }^{\mathbb{R}}$ ), either FLrFVIII or BDDrFVIII, were investigated by 1- and 2-DE and MS. The objective of this study was to compare the heterogeneity and the high purity of both rFVIII preparations before and after thrombin digestion. In particular, the 2-D gel was optimized to better highlight the presence of contaminants and many unexpected proteins. Recombinant strategies consisting of insertion of expression vectors containing BDDrFVIII and FLrFVIII resulted in homogeneous and heterogeneous protein products, respectively, the latter consisting in a heterogeneous mixture of various B-domain-truncated forms of the molecule. Thrombin digestion of all the three rFVIII gave similar final products, plus one unexpected fragment of A2 domain missing 11 amino acids. Regarding the contaminants, Helixate NexGen ${ }^{\circledR}$ showed the presence of impurities, such as Hsp70 kDa, haptoglobin and proapolipoprotein; Refacto ${ }^{\circledR}$ showed glutathione $S$-transferase and $\beta$-lactamase, whereas Advate ${ }^{\circledR}$ apparently did not contain any contaminants. The proteomic approach will contribute to improving the quality assurance and manufacturing processes of rFVIII concentrates. In this view, the 2-DE is mandatory for revealing the presence of contaminants.
\end{abstract}

\section{Keywords:}

2-DE / Hemophilia A / Proteomics / Recombinant factor VIII

DOI 10.1002/elps.201000216

\section{Introduction}

Hemophilia A (factor VIII deficiency) is an X-linked, recessive, bleeding disorder caused by heterogeneous mutations in the FVIII gene, which affects one in 10000 individuals (one in 5000 males) in most populations. Human clotting factor VIII (FVIII) is a non-enzymatic plasma glycoprotein that plays an essential role in the intrinsic pathway of the blood coagulation cascade [1]. The degree of FVIII deficiency correlates with the frequency of clinically significant bleeding [2]. Two categories of FVIII concentrates are commercially available, namely plasma-

Correspondence: Dr. Anna Maria Timperio, Tuscia University, Largo dell' Università snc, 01100 Viterbo, Italy

E-mail: timperio@unitus.it

Fax: +39-0761-357179

Abbreviations: CHO, Chinese hamster ovary; FA, formic acid derived or recombinant FVIII: the former is extracted from human blood, while the latter is biosynthesized through recombinant DNA technology. Infusion of both plasmaderived and/or recombinant factor VIII (rFVIII) is the standard strategy for treating hemorrhage in patients with hemophilia A.

Native protein is synthesized as a single polypeptide chain of 2332 amino acids, which is preceded by a 19-residue hydrophobic signal peptide of importance for the secretion. The protein contains six main domains: three Atype domains, a central B domain and two C-type domains. In detail, domains can be distinguished as follows: three small acidic peptide regions ( 30-40 designed by "a") join (i) the A1 and A2 domains (residues 337-372); (ii) the A2 and B domains (residues 711-740); and (iii) the last one connects the $\mathrm{B}$ domain with the amino terminal of the A3 domain (residues 1649-1689). Therefore, the domain

\footnotetext{
${ }^{*}$ These authors contributed equally to this work and share the first authorship.
} 